\title{
Knowledge, perceived barriers and facilitators of medication error reporting: a quantitative survey in Malaysian primary care clinics
}

\author{
A. Samsiah ${ }^{1} \cdot$ Noordin Othman $^{2,3} \cdot$ Shazia Jamshed $^{4,5} \cdot$ Mohamed Azmi Hassali $^{6}$
}

Received: 7 May 2019 / Accepted: 16 April 2020 / Published online: 3 June 2020

(c) Springer Nature Switzerland AG 2020

\begin{abstract}
Background Medication errors are the most common types of medical errors that occur in health care organisations; however, these errors are largely underreported. Objective This study assessed knowledge on medication error reporting, perceived barriers to reporting medication errors, motivations for reporting medication errors and medication error reporting practices among various health care practitioners working at primary care clinics. Setting This study was conducted in 27 primary care clinics in Malaysia. Methods A self-administered survey was distributed to family medicine specialists, doctors, pharmacists, pharmacist assistants, nurses and assistant medical officers. Main outcome measures Health care practitioners' knowledge, perceived barriers and motivations for reporting medication errors. Results $\mathrm{Of}$ all respondents $(\mathrm{N}=376)$, nurses represented $31.9 \%(n=120)$, followed by doctors $(n=87,23.1 \%)$, pharmacists $(n=63,16.8 \%)$, assistant medical officers $(n=53,14.1 \%)$, pharmacist assistants $(n=46,12.2 \%)$ and family medicine specialists $(n=7,1.9 \%)$. Of the survey respondents who had experience reporting medication errors, $56 \%(n=62)$ had submitted medication error reports in the preceding 12 months. Results showed that $41.2 \%(n=155)$ of respondents were classified as having good knowledge on medication error and medication error reporting. The mean score of knowledge was significantly higher among prescribers and pharmacists than nurses, pharmacist assistants and assistant medical officers $(p<0.05)$. A heavy workload was the key barrier for both nurses and assistant medical officers, while time constraints prevented pharmacists from reporting medication errors. Family medicine specialists were mainly unsure about the reporting process. On the other hand, doctors and pharmacist assistants did not report primarily because they were unaware medication errors had occurred. Both family medicine specialists and pharmacist assistants identified patient harm as a motivation to report an error. Doctors and nurses indicated that they would report if they thought reporting could improve the current practices. Assistant medical officers reported that anonymous reporting would encourage them to submit a report. Pharmacists would report if they have enough time to do so. Conclusion Policy makers should consider using the information on identified barriers and facilitators to reporting medication errors in this study to improve the reporting system to reduce under-reported medication errors in primary care.
\end{abstract}

Keywords Barriers $\cdot$ Facilitators $\cdot$ Knowledge $\cdot$ Malaysia $\cdot$ Medication error reporting $\cdot$ Primary care

Noordin Othman

noordin_uia@yahoo.com

1 Institute for Health Systems Research, Ministry of Health, 40170 Shah Alam, Selangor, Malaysia

2 Department of Clinical and Hospital Pharmacy, College of Pharmacy, Taibah University, Almadinah, Almunawwarah 30001, Kingdom of Saudi Arabia

3 Faculty of Pharmacy, PICOMS International University College, No 3, Jalan 31/10A, Taman Batu Muda, 68100 Batu Caves, Kuala Lumpur, Malaysia
4 Kuliyyah of Pharmacy, International Islamic University Malaysia, 25200 Kuantan, Pahang, Malaysia

5 Qualitative Research-Methodological Applications in Health Sciences Research Group, Kuliyyah of Pharmacy, International Islamic University Malaysia, 25200 Kuantan, Pahang, Malaysia

6 School of Pharmaceutical Sciences, Universiti Sains Malaysia, 11800 Gelugor, Penang, Malaysia 


\section{Impacts on practice}

- Identification of key barriers and avenues to medication error reporting should facilitate the development and improvement of a more effective and efficient medication error reporting system.

- Given the importance of improving medication delivery in the healthcare system, more education and training programmes on the error reporting process are needed for all healthcare practitioners in primary care clinics involved in the medication use cycle, including learning to report near misses.

- Lessons learnt from reported medication errors should be translated into improving prescribing and dispensing processes and practices to encourage more active reporting from healthcare practitioners.

\section{Introduction}

The National Coordinating Council for Medication Error Reporting and Prevention (NCC MERP) has defined medication errors (MEs) as "any preventable event that may cause or lead to inappropriate medication use or patient harm while the medication is in the control of the health care professional, patient, or consumer" [1]. This definition also includes near misses or potential errors, which fall under category A and B using the NCC MERP classification system.

MEs are the most common types of medical errors that occur across healthcare organisations; however, in many countries, they are mostly underreported. Errors indicate a problem within a system, which warrants investigation; an error reporting system allows healthcare organisations to mitigate the contributing factors, prevent recurrence of errors, and learn from the errors [2, 3]. However, failure to report MEs limits the development of prevention strategies.

Error reporting is a voluntary process. Therefore, underreporting poses a significant challenge. Several barriers to error reporting as well as motivations to report errors have been previously described: numerous studies cited fear as a major barrier to error reporting, either in hospitals [4-9], primary care settings [10-12] or nursing homes $[13,14]$. The time constraints was another inhibitor of reporting $[4,8,9,11,14-16]$. Additionally, lack of feedback to reporters or organisations hinders reporting $[5$, $6,9,14,17-20]$. Furthermore, errors resulting in no harm discourages reporting $[4,5,8,14,15,18]$.

In contrast, healthcare practitioners (HCPs) are highly likely to report errors associated with severe outcomes $[8$,
$15,21]$. Clear reporting procedures encourages reporting $[8,15,19,20]$. In addition, learning from past mistakes $[6,17,22]$ and errors has led to improvement of current practices $[8,22]$, compelling HCPs to increase reporting.

Factors unique to a particular healthcare setting that act as barriers to ME reporting need to be addressed and understood, and effective ways to remove them should be implemented. Equally important, efforts should also be directed at exploring factors that encourage reporting. Globally, research on ME reporting mostly focuses on secondary care, while the primary care setting has been neglected.

\section{Aim of the study}

This study aims to assess the knowledge of ME reporting, barriers to ME reporting, main facilitators for ME reporting as well as ME reporting practices among various HCPs in selected primary care clinics in Malaysia.

\section{Methods}

\section{Design}

This is a cross-sectional study conducted from March to May 2016, using a validated questionnaire. HCPs working at selected primary care outpatient clinics under the Ministry of Health Malaysia (MoH) were involved in the study.

\section{Participants}

Four states were involved, which were conveniently sampled to represent four regions in Peninsular Malaysia. A district was selected in each state, and all primary care clinics under the $\mathrm{MoH}$ listed under each district were then included as the target study sites. A total of 27 clinics were involved in this study.

The target population were the HCPs: family medicine specialists (FMSs), doctors, pharmacists, pharmacist assistants (PAs), nurses, and assistant medical officers (AMOs), with an estimated total number of approximately 14,000 potential respondents. A response from 374 HCPs was required to yield a margin of error of $5 \%$ and confidence interval of $95 \%$. A total of 462 questionnaires were distributed, taking into consideration approximately a $20 \%$ nonresponse rate.

The total number of samples for each category of HCPs in each clinic was determined by proportion, and the potential respondents were randomly drawn from a full list of the target population in each clinic obtained from the respective district health office. However, all FMSs were included because of their small number in every district (one or two 
per district). Due to this small number, FMSs and doctors' group were combined as a prescriber group for ANOVA test.

\section{Questionnaire development and distribution}

The questionnaire was constructed following a comprehensive review of literature and was based on the clinical experience of the researchers. A series of in-depth interviews exploring knowledge, barriers, facilitators and reporting practice was conducted among a purposely sampled group of similar HCPs to support the questionnaire development process [23]. Findings from the interviews were incorporated into the drafted questionnaire. Three clinical pharmacy experts, highly experienced in ME reporting practice and research in Malaysia, assessed the content validity of the questionnaire's individual items to verify its importance and relevance. The content validity index for individual items (I-CVI) was 0.94, and the content validity index for scales (S-CVI) was also 0.94, indicative of the questionnaire's good content validity. Prior to this survey, the questionnaire was validated with a convenience sample of 175 respondents who were excluded from this study. The validity and reliability of the questionnaire were assessed. A Cronbach's alpha value of greater than 0.7 for all items (knowledge, barriers, facilitators) indicates good internal consistency. The correlation coefficients for all scales of knowledge were fair to good (ICC exceeded 0.4) and more than 0.7 for barriers and facilitators supported the instrument reliability.

The questionnaire consisted of five parts:

i. Respondents' background (eight questions);

ii. Knowledge on ME reporting (22 questions) which applied "true/false" options;

iii. Barriers to reporting MEs (23 questions divided into five main categories: disagreement about errors, reporting effort, concern-related factors, administrative/organisation and working environment);

iv. Facilitators for reporting ME (14 questions divided into four main categories: errors, monitoring, administrative, provider-related);

v. ME reporting practices (two questions on experience with reporting MEs)

Questions on barriers and facilitators used a 5-point Likert scale, with responses ranging from 1 - strongly disagree to 5 - strongly agree.

The questionnaires were mailed to the respondents through the pharmacist of each clinic, who acted as a mediator. The pharmacist distributed the forms, reminded the respondents to fill in the forms, and gathered the completed questionnaires. The compiled questionnaires were then collected personally by the main researcher.

\section{Data analysis}

Data was checked for completeness, coded, and entered into a database developed in Microsoft Access. Completed data was then transferred to SPSS version 21 for analysis. Frequencies and percentages (\%) were produced for the respondents' characteristics. The total knowledge score was calculated from part ii of the questionnaire by giving one point (1) for each correct answer and zero (0) for incorrect answers or for no response. Respondents who scored $\leq 12$ were grouped as low knowledge, those with a score of $13-20$ as having fair knowledge and those with a score $\geq 21$ as having good knowledge on ME and ME reporting. The key barriers to ME reporting and facilitators for reporting were indicated by category and individual statement using mean scores.

An ANOVA was used to test the significant differences between professional groups (prescribers, pharmacists, pharmacist assistants, nurses, and AMOs). A post hoc test was used as comparison procedure if an ANOVA showed a significant difference. A value of $p<0.05$ was considered to be statistically significant.

\section{Ethics approval}

The Medical Research and Ethics Committee Ministry of Health Malaysia (NMRR-13-1219-17743) and International Islamic University of Malaysia Research Ethic Committee (IREC 162) approved this study. Permission was obtained from the respective directors of the Health State Department and the District Health Office. The survey questionnaire was enclosed with a cover letter briefly describing the survey and a consent statement. Completing and returning the questionnaire served to signify the respondent's voluntary consent.

\section{Results}

\section{Respondents' characteristics}

A total of 376 respondents returned the completed questionnaires, yielding a response rate of $81.4 \%$ (Table 1). Women constituted the majority of respondents (74.8\%). About half of the respondents $(52.1 \%)$ were in the age group of 25 to 34 years old. Nurses represented $31.9 \%$ of total respondents, followed by doctors (23.1\%) and pharmacists (16.8\%). Approximately $35 \%$ of respondents had five years or fewer of working experience. Almost $65 \%$ of the respondents had not attended any formal training on ME reporting during their working tenancy. 
Table 1 Respondent Characteristics

\begin{tabular}{|c|c|c|}
\hline Characteristics & $\begin{array}{l}\text { Frequency } \\
(\mathrm{n}=376)\end{array}$ & $\%$ \\
\hline \multicolumn{3}{|l|}{ Age group, years } \\
\hline$<25$ & 19 & 5.1 \\
\hline $25-34$ & 196 & 52.1 \\
\hline $35-44$ & 102 & 27.1 \\
\hline $45-54$ & 48 & 12.8 \\
\hline$\geq 55$ & 11 & 2.9 \\
\hline \multicolumn{3}{|l|}{ Sex } \\
\hline Male & 91 & 24.2 \\
\hline Female & 285 & 75.8 \\
\hline \multicolumn{3}{|l|}{ Ethnicity } \\
\hline Malay & 305 & 81.1 \\
\hline Chinese & 45 & 12.0 \\
\hline Indian & 23 & 6.1 \\
\hline Others & 3 & 0.8 \\
\hline \multicolumn{3}{|l|}{ Profession } \\
\hline FMS & 7 & 1.9 \\
\hline Doctor & 87 & 23.1 \\
\hline Pharmacist & 63 & 16.8 \\
\hline PA & 46 & 12.2 \\
\hline Nurse & 120 & 31.9 \\
\hline AMO & 53 & 14.1 \\
\hline \multicolumn{3}{|c|}{ Total Duration of service (years) } \\
\hline$\leq 5$ & 129 & 34.3 \\
\hline $6-10$ & 107 & 28.5 \\
\hline $11-15$ & 64 & 17.0 \\
\hline $16-20$ & 34 & 9.0 \\
\hline$\geq 21$ & 42 & 11.2 \\
\hline \multicolumn{3}{|c|}{ Attended training on ME reporting } \\
\hline Yes & 121 & 32.2 \\
\hline No & 243 & 64.6 \\
\hline Unknown (missing) & 12 & 3.2 \\
\hline
\end{tabular}

\section{ME reporting practice}

More than two-thirds of the respondents (70\%) stated that they had never reported any ME. Of those who had experienced reporting MEs, only $56 \%$ submitted ME reports in the preceding 12 months (Table 2$)$, with $82.3 \%(n=51)$ reporting five or fewer MEs, $8 \%(n=5)$ reporting 6 to 10 MEs and $9.7 \%(n=6)$ reporting 10 MEs or more.

\section{Knowledge on ME reporting}

Of all participants $(n=376), 41.2 \%(n=155)$ were classified as having a good knowledge of ME and ME reporting, scoring $\geq 21$ on the ME knowledge portion of the survey. Based on individual statements, $25.8 \%(\mathrm{n}=97)$ respondents indicated that MEs were unpreventable, $40.4 \%(n=152)$ were
Table 2 ME reporting practices

\begin{tabular}{lcc}
\hline Profession & $\begin{array}{l}\text { Number of ever } \\
\text { reported ME (\%) } \\
\mathrm{n}=111\end{array}$ & $\begin{array}{l}\text { Number of } \\
\text { reported ME } \\
\text { in the past } \\
12 \text { months } \\
(\%) \\
\mathrm{n}=62\end{array}$ \\
\hline FMS & $5(4.5 \%)$ & $2(3.22 \%)$ \\
Doctor & $31(27.93 \%)$ & $17(27.42 \%)$ \\
Pharmacist & $43(38.74 \%)$ & $25(40.32 \%)$ \\
PA & $14(12.61 \%)$ & $8(12.90 \%)$ \\
Nurse & $14(12.61 \%)$ & $7(11.30 \%)$ \\
AMO & $4(3.61 \%)$ & $3(4.84 \%)$ \\
\hline
\end{tabular}

unaware that near misses require reporting, 26.9\% $(n=101)$ assumed that omission errors should not be reported, and 47.9\% $(n=180)$ did not know that the Medication Error Reporting System (MERS) is applicable in healthcare facilities other than those under the MoH. However, the majority (greater than $80 \%$ ) of the respondents answered correctly on questions on who should report MEs and what system(s) should to be used to report ME.

There was a statistically significant difference in the total knowledge score of the professionals' group, $\mathrm{F}$ $(4,371)=9.023, p<0.05$. A Tukey post hoc test revealed that the prescribers group had a higher knowledge score compared to the nurses' group $(1.45 \pm 0.312 ; p=0.000)$, PAs $(1.34 \pm 0.408 ; p=0.010)$, and AMOs $(1.10 \pm 0.390$; $p=0.039)$. A Tukey post hoc test further revealed that the pharmacists group had a higher knowledge score compared with the nurses' group $(1.460 \pm 0.353 ; p=0.000)$, PAs $(1.50 \pm 0.440 ; p=0.006)$, and AMOs $(1.26 \pm 0.423$; $p=0.025)$.

\section{Barriers to ME reporting}

Of all respondents $(n=376)$, the main perceived barriers were in the category of reporting effort (mean $=3.68$; $\mathrm{SD}=0.65$ ), followed by organisation/administrative barriers $($ mean $=3.46 ; \mathrm{SD}=0.56$ ), the working environment $($ mean $=3.31 ; \mathrm{SD}=0.86)$, concern-related factors $($ mean $=3.26 ; \mathrm{SD}=0.76)$, and disagreement over errors (mean $=3.02 ; \mathrm{SD}=0.84$ ).

The top three individual barriers (Table 3) were heavy workloads, time constraints, and unclear reporting processes. Table 4 highlights the key barriers to reporting MEs as perceived by each professional group.

Significant differences were found among the professional's group for two key barriers: heavy workloads and time constraints, $\mathrm{F}(4,371)=2.416, p=0.048$ and $\mathrm{F}$ $(4,371)=3.526, p=0.008$, respectively. A Tukey post hoc test revealed that pharmacists' group were statistically more 
Table 3 Barriers to reporting MEs (top 10)

\begin{tabular}{|c|c|c|c|c|c|c|}
\hline Rank & Category & Item & Mean & SD & $\mathrm{F}$ & Sig-F \\
\hline 1 & Reporting effort & Heavy workloads & 3.90 & 0.87 & 2.416 & 0.048 \\
\hline 2 & Reporting effort & Time constraints & 3.85 & 0.85 & 3.526 & 0.008 \\
\hline 3 & $\begin{array}{l}\text { Organisation/ } \\
\text { Administrative }\end{array}$ & $\begin{array}{l}\text { Lack of understanding of the process of reporting (e.g., what to report, } \\
\text { how to make a report, who is responsible for reporting it) }\end{array}$ & 3.82 & 0.82 & 0.378 & 0.825 \\
\hline 4 & Reporting effort & Additional paperwork following reporting & 3.74 & 0.87 & 1.269 & 0.282 \\
\hline 5 & Disagreement over error & Unaware medication error has occurred & 3.73 & 0.96 & 5.665 & 0.000 \\
\hline 6 & $\begin{array}{l}\text { Organisation/ } \\
\text { Administrative }\end{array}$ & Lack of promotion to encourage reporting & 3.70 & 0.80 & 0.986 & 0.415 \\
\hline 7 & $\begin{array}{l}\text { Organisation/ } \\
\text { Administrative }\end{array}$ & Lack of feedback from reporting & 3.66 & 0.76 & 0.309 & 0.872 \\
\hline 8 & $\begin{array}{l}\text { Organisation/ } \\
\text { Administrative }\end{array}$ & Lack of enforcement or emphasis on reporting & 3.65 & 0.82 & 0.485 & 0.747 \\
\hline 9 & Concern-related factors & Concern of repercussion by a higher authority & 3.56 & 1.10 & 6.700 & 0.000 \\
\hline 10 & Reporting effort & System or form used for reporting medication error is time-consuming & 3.53 & 0.92 & 0.715 & 0.582 \\
\hline
\end{tabular}

Table 4 Main perceived barriers and facilitators to ME reporting (by professional group)

\begin{tabular}{lllll}
\hline Professional group & Barriers & Mean (SD) & Facilitators & Mean (SD) \\
\hline FMS & Unclear reporting process & $3.86(1.06)$ & Error harmed patient & $4.71(0.49)$ \\
Doctors & Unaware error occurred & $4.02(0.85)$ & Reporting improve practice & $4.20(0.66)$ \\
Pharmacists & Time constraints & $4.19(0.76)$ & Ample time & $4.35(0.86)$ \\
PA & Unaware error occurred & $3.78(0.63)$ & Error harmed patient & $4.02(0.72)$ \\
AMO & Heavy workload & $3.98(0.77)$ & Anonymous reporting & $4.02(0.80)$ \\
Nurses & Heavy workload & $3.95(0.84)$ & Reporting improve practice & $4.16(0.52)$ \\
\hline
\end{tabular}

likely to indicate that heavy workload as a barrier to ME reporting than the PAs' group $(0.465 \pm 0.167, p=0.045)$. Additionally, pharmacists' group had statistically higher agreement that time constraints deterred them from reporting compared with nurses' group $(0.440 \pm 0.131, p=0.007)$ and PAs' group $(0.517 \pm 0.163, p=0.014)$.

Being unaware that a ME has occurred also was significantly different among the groups, $\mathrm{F}(4,371)=5.665$, $p=0.01$. This barrier was statistically higher in the prescribers' group compared with the nurses' group $(0.583 \pm 0.13$, $p=0.000$ ), and higher in pharmacists than in the nurses' group $(0.456 \pm 0.146, p=0.017)$.

The third significant difference in perceived barriers among the groups was concern of repercussion by a higher authority, $\mathrm{F}(4,371)=6.700, p=0.00$. Nurses $(0.826 \pm 0.166$, $p=0.000)$, AMOs $(0.701 \pm 0.199, p=0.004)$, and PAs groups $(0.571 \pm 0.207, p=0.047)$ were statistically more likely to indicate concern of repercussion by a higher authority as a barrier to ME reporting compared with the pharmacist's group.

\section{Facilitators for ME reporting}

Overall, the respondents showed a high level of agreement for the statements in all dimensions of facilitators, with a mean of 3.88 and greater in each category. The major perceived facilitators were in the dimension of error (mean 4.01; $\mathrm{SD}=0.63)$, provider-related factors $($ mean $=3.94$; $\mathrm{SD}=0.55)$, administrative factors ( mean $=3.90 ; \mathrm{SD}=0.61)$, and monitoring factors (mean $=3.88 ; \mathrm{SD}=0.61$ ).

Based on individual statements (Table 5), the most commonly perceived facilitators of ME reporting were belief of reporting can improve current practices, errors harm patients, and training HCPs in using the reporting system can mitigate errors. Further analysis on the main facilitator perceived by each professional group is shown in Table 4 .

There was a statistically significant difference among professionals' groups for the facilitator statement, "Healthcare provider is trained to use the reporting system," $\mathrm{F}$ $(4,371)=4.059, p=0.003$. A Tukey post hoc test revealed that pharmacists' group were statistically more likely to perceive this factor as motivation to reporting compared with the PAs' group $(0.359 \pm 0.126, p=0.038)$ and nurses' group $(0.296 \pm 0.101, p=0.036)$.

Statistically significant difference was also observed among different professional groups for the statement, "If there is enough time to report"; $\mathrm{F}(4,371)=6.592, p=0.000$. A Tukey post hoc test showed pharmacists' group were statistically more likely to belief that this factor encouraged reporting compared with the PAs' group $(0.545 \pm 0.151$, 
Table 5 Facilitators for ME reporting

\begin{tabular}{|c|c|c|c|c|c|c|}
\hline Rank & Category & Item & Mean & SD & $\mathrm{F}$ & Sig-F \\
\hline 1 & Administrative & $\begin{array}{l}\text { If reporting can improve practices (e.g. prescribing quality, } \\
\text { dispensing quality, etc.) }\end{array}$ & 4.15 & 0.58 & 2.439 & 0.05 \\
\hline 2 & Error & Patient was harmed & 4.03 & 0.72 & 0.079 & 0.99 \\
\hline 3 & Provider-related & If healthcare provider is trained to use the reporting system & 4.02 & 0.66 & 4.059 & 0.00 \\
\hline 4 & Error & If ME involves medico- legal issues & 4.01 & 0.73 & 1.796 & 0.13 \\
\hline 5 & Provider-related & As self-protection if any issue arises later & 4.00 & 0.68 & 1.345 & 0.25 \\
\hline 6 & Error & If the same error is repeated & 3.98 & 0.73 & 0.567 & 0.69 \\
\hline 7 & Provider-related & If there is enough time to report & 3.96 & 0.80 & 6.592 & 0.00 \\
\hline 8 & Monitoring & If there is appropriate monitoring & 3.95 & 0.66 & 3.770 & 0.10 \\
\hline 9 & Monitoring & If reporting of medication errors is promoted & 3.93 & 0.74 & 3.290 & 0.01 \\
\hline 10 & Administrative & If I know that I will get feedback on the reporting status & 3.87 & 0.74 & 1.277 & 0.28 \\
\hline
\end{tabular}

$p=0.003)$, the nurses' group $(0.583 \pm 0.121, p=0.000)$ and the AMOs' group $(0.425 \pm 0.145, p=0.030)$.

More awareness and promotion on the importance reporting of ME was another facilitator that was found statistically significant difference among different groups, F $(4,371)=3.290, p=0.011$. A Tukey post hoc test revealed that perception of this facilitator was statistically higher in the prescribers' group compared with the AMOs' group $(0.363 \pm 0.125, p=0.033)$, and higher in the pharmacists' group than the AMOs' group $(0.416 \pm 0.136, p=0.020)$.

\section{Discussion}

Earlier studies on this topic mostly examined perceptions and attitudes towards ME reporting among nurses [7, 24-26], nurses or/and physicians [4-6, 19, 20] or physicians, nurses, and pharmacists $[8,27,28]$. This study, however, goes to a greater extent; multiple HCPs involved in the medication use cycle in primary care clinics, namely, doctors, nurses, pharmacists, PAs, and AMOs were included. Participation of multiple HCPs is essential as they share equal responsibility for delivering safe drug treatment to patients. Identification of measures to improve reporting is very crucial and should be based on barriers and facilitators perceived by HCPs, taking into consideration related behaviour change theories [9].

\section{ME reporting practice}

A medication error reporting system is effective and efficient when it enables and encourages HCPs to report any types of ME irrespective of the severity and near misses [29]. These reports help facilitate early identification of patient safety issues [30]. Hence, our findings that more than twothirds of the respondents had never reported any ME during their working tenure and that only half of those who had experienced reporting ME had submitted ME reports in the preceding 12 months showed that underreporting MEs is prevalent. This situation prevents better understanding of the root causes of the problem, thus reducing opportunities to learn from past mistakes and act on ME reports.

Similar to findings from other studies $[6,31,32]$, this study observed that ME reporting among respondents was low $(30 \%)$, an indication of underreporting and yet a prominent culture in primary care clinics. Pharmacists were the prime ME reporters among HCPs who reported MEs in the past 12 months, perhaps an indication that the existing medication error reporting system is mostly known by the pharmacy staff.

\section{Knowledge on ME reporting}

Knowledge of ME reporting is fundamental to ensure HCPs report every error. To accomplish this, HCPs must be cognisant of the ME reporting system at their workplace, and the reporting workflow should be explicitly clear, starting with identification of reportable events, what form to be used, who should report, and to whom the error report should be submitted to.

In this study, we found that prescriber and pharmacist groups have higher knowledge of ME reporting than nurses, PAs, and AMOs. A similar result was found in a study among physicians, pharmacists, and nurses in hospitals in Manila; the pharmacists were found to be the most knowledgeable in ME reporting [28]. This may imply that both prescribers and pharmacists are well-versed in matters related to ME reporting and should assist other HCPs. Pharmacists, who are more knowledgeable in this aspect with solid basic knowledge on drugs, should play a bigger role in promoting and engaging other HCPs in ME reporting. Near misses occur 10-300 times more frequently than actual events and offer the opportunity to correct the system before ME cause serious harm [30]. However, this study also found that about $40 \%$ of the respondents were uncertain about the 
need to report near misses, and approximately $30 \%$ believed that omission errors do not require reporting. This indicates uncertainty around what constitutes a reportable ME, which need to be addressed and emphasised during training and education [33]. In addition, reporting near misses yields positive effects since the events did not cause any harm to the patient; thus, the reporter will not be blamed. Instead, such report should be commended as it prevented injury or harm to the patient [34]. Therefore, in the effort to improve patient safety, healthcare organisation should highlight on the importance of learning from what works as well as from what fails in the healthcare systems. This will help the organisation to better understand how things go right, proactively and continuously trying to anticipate future events [33].

\section{Perceived barriers to ME reporting}

Our findings suggest that heavy workloads, time constraints, and lack of certainty around the ME reporting process influences the willingness of HCPs to report MEs. Several earlier studies in primary care settings highlighted similar findings, indicating workload $[10,12]$ and time constraints $[10,12$, 35-38] as being among the obvious barriers to error reporting. Numerous studies from other settings have confirmed these findings $[8,15,16,18-20,31,39-41]$; however, those results contradicted a recent local study among doctors and pharmacists in primary care clinics, which found that workload did not interfere with the respondents' ability to report MEs [42].

Unclear reporting processes (i.e. what to report, how to make a report, who is responsible for reporting) was also deemed to be another major hurdle to ME reporting in this study. Analysis of ME knowledge revealed that the majority of respondents knew who should report ME and were familiar with the reporting system. However, emphasis should be given to clarifying the definition of reportable events, specifically near misses and omission errors; the percentage of respondents who misunderstood these matters was reasonably high. Omission errors or the failure to administer an ordered dose to a patient before the next scheduled dose, if any, was among the most frequently reported types of errors in previous research [43-47]. However, findings from a local study based on a review of MEs reported to the National Medication Error Reporting System showed that omitted or delayed medicines occurred in $4.2 \%$ of total reports received, which may be due to uncertainty regarding whether such errors should be reported [48]. Lack of familiarity with the reporting process was another reason for not reporting, as revealed by other primary care research study $[11,35,49$, 50]. Similarly, this factor was also prominent in other healthcare settings $[8,14,16,19,20,22,51-53]$.
Another important observation of this study is that none of the statements regarding concern-related factors or fear were among the top five barriers to reporting, contradicting previous international studies. However, fear of repercussion by a higher authority (e.g. disciplinary action or legal action) were prominent among nurses, AMOs and PAs compared to prescribers and pharmacists. This is consistent with previous studies of nurses in hospital settings [4, 5, 7, 24, 31, 54-58] and a study among nurses in community health service [50], who cited fear-related factors as a major obstacle to ME reporting. A possible explanation could be that the relative hierarchy and social influence between groups within a healthcare organisation deter reporting doctors' or pharmacists' errors by these groups. As frontline staff, nurses, AMOs and PAs are concerned with the consequences of reporting - disciplinary or legal actions - due to their direct involvement with error as well as the possibility of being blamed [4]. These are complex issues and highly dependent on the culture of each organisation. Previous literature has identified the most common definition of safety culture as, "the product of individual and group values, attitudes, competencies and patterns of behaviour that determine the commitment to, and the style and proficiency of, an organisation's health and safety programmes" [59]. Therefore, healthcare organisations should aim to provide a positive safety culture in which HCPs feel safe to report and discuss errors [59], ultimately promoting learning from the incidents to improve patient safety.

This argument is supported by Azjen and Madden's Theory of Planned Behaviour, which outlines three factors determining HCPs intentions to report an incident: i) perceived behavioural control, which measures internal and external resources (ability, expertise, knowledge, time and cooperation); ii) perception of social expectation of incident reporting; and iii) general attitudes and personal beliefs about incident reporting. In a study examining this theory among community nurses [57], the authors concluded that the most meaningful factors affecting the intention to report an adverse incident are the nurse's perceived behavioural control and her perception of what her social and professional community expect of her with regard to adverse incident reporting. In other words, the nurse needs a positive organisational culture that supports incident reporting in addition to adequate resources including support, knowledge, and time that will assist her in performing incident reporting.

\section{Perceived facilitators for ME reporting}

Interestingly, all groups of respondents considered improvement to current practice including prescribing and dispensing quality as a key motivator for ME reporting. Other studies made similar findings [6,17,22, 31], observing that this 
denotes respondents' desire to see actual positive changes based on the reported events, reflecting a mutual commitment to improve safety measures for medication use among both the management and the healthcare professionals at the clinics. Sufficient evidence of the reporting outcome linked with noticeable changes and sustainable action in the system/organisation is an indication that the reporting system has achieved its target [3].

An error that caused harm to a patient increases the respondent's likelihood to report the ME. Examining attitudes regarding ME reporting indicated that HCPs have a better positive attitude towards reporting harmful ME. Our findings also indicated that patient safety with regards to ME is the top priority of HCPs, consistent with other studies suggesting that HCPs reported serious incidents or if their patients' outcomes were bad $[5,8,14,15,18,60]$.

HCPs well trained in using the current ME reporting system was another facilitator for ME reporting in this study. This includes the reporting process - identification of a reportable event, determination of what form or mechanism of reporting should be used, and identification of who should report the error. HCPs acknowledged and valued the importance of training on the reporting process to ensure they can fully utilise the reporting system. In addition, earlier studies emphasised the importance of training HCPs to use the reporting mechanism to increase reporting $[8,19,20]$.

\section{Strengths and limitation}

This study was conducted using a validated questionnaire, which has been tested within the same context of the actual survey. A high response rate was one of the strengths of this study. This may be due to high HCP interest in a topic highly relevant to their patients' safety, which is a main concern in delivering a quality healthcare.

Another strong point of this study is the participation of multiple HCPs groups directly involved in medication safety as the target population. This provides researchers with a chance to gain better insight into the problems being studied.

On the other hand, this study is not without limitation. We noted the small sample size of the FMSs who took part in this survey which was due to the small number of FMSs in each district/state and the feasibility issue of including all FMSs in the country. Therefore, we had combined them with the doctors in our analysis.

The questionnaire provides no open field questions for respondents to provide further comments. However, this limitation was minimised by incorporating findings from a series of interviews conducted among a similar group of HCPs during the questionnaire development process into the drafted questionnaire.

Since this study was carried out in primary care clinics, the results might not be generalisable to other healthcare settings. There was also a potential for recall bias, when the respondents were asked on their reporting experience and the number of error reports submitted in the past 12 months.

\section{Conclusion}

This study quantified the key barriers and facilitators to medication error reporting according to the five main health care practitioners in primary care clinics involved in the medication use process. Findings from this study may be used to develop and/or improve effectiveness and efficiency of medication error reporting systems, impacting patient safety. Perceived barriers were mainly centred on issues on reporting effort and knowledge of the reporting process, while respondents showed high agreement on four categories of facilitators: dimension of error, provided-related factors, administrative factors and monitoring factors.

Acknowledgements We would like to thank the Director General of Health Malaysia for his approval to publish this work. We thank the Health State Departments and Health District Offices for their permission to conduct this study in their respective states and clinics. We extend our appreciations to the pharmacists who assisted in the data collection and all the respondents who participated in this study.

Funding This research received no funding from any agency.

Conflicts of interest None.

\section{References}

1. NCCMERP. The National Coordinating Council for Medication Error Reporting and Prevention NCC MERP : The First Ten Years " Defining the Problem and Developing Solutions ". 2005.

2. Leistikow I, Mulder S, Vesseur J, Robben P. Learning from incidents in healthcare: the journey, not the arrival, matters. BMJ Qual Saf. 2017;26:252-6.

3. Mitchell I, Schuster A, Smith K, Pronovost P, Wu A. Patient safety reporting : a qualitative study of thoughts and perceptions of experts 15 years after ' To Err is Human'. BMJ Qual Saf. 2016;25:92-9.

4. Yung H, Yu S, Chu C, Hou I, Tang F. Nurses' attitudes and perceived barriers to the reporting of medication administration errors. J Nurs Manag. 2016;24:580-8.

5. Abualrub RF, Al-akour NA, Alatari NH. Perceptions of reporting practices and barriers to reporting incidents among registered nurses and physicians in accredited and nonaccredited Jordanian hospitals. J Clin Nurs. 2015;24:2973-82.

6. Rashed A, Hamdan M. Physicians' and nurses' perceptions of and attitudes toward incident reporting in Palestinian Hospitals. J Patient Saf. 2015;00:1-6.

7. Bayazidi S, Zarezadeh Y, Parvan K. Medication error reporting rate and its barriers and facilitators among nurses. J Caring Sci. 2012;1:231-6.

8. Hartnell N, MacKinnon N, Sketris I, Fleming M. Identifying, understanding and overcoming barriers to medication error reporting in hospitals: A focus group study. BMJ Qual Saf. 2012;21:361-8. 
9. Alqubaisi M, Tonna A, Strath A, Stewart D. Exploring behavioural determinants relating to health professional reporting of medication errors: a qualitative study using the Theoretical Domains Framework. Eur J Clin Pharmacol. 2016;72:887-95.

10. Escoto KH, Karsh B-T, Beasley JW. Multiple user considerations and their implications in medical error reporting system design. Hum Factors. 2006;48:48-58.

11. Hickner J, Zafar A, Kuo GM, Fagnan LJ, Forjuoh SN, Stevens $\mathrm{BK}$, et al. Field test results of a new ambulatory care medication error and adverse drug event reporting- MEADERS. Ann Fam Med. 2010;8:517-25.

12. Rea D, Griffiths S. Patient safety in primary care: incident reporting and significant event reviews in British general practice. Health Soc Care Community. 2016;24:411-9.

13. Wagner LM, Damianakis T, Pho L, Tourangeau A. Barriers and facilitators to communicating nursing errors in long term care settings. J Patient Saf. 2013;9:1-7.

14. Handler SM, Perera S, Olshansky EF, Studenski SA, Nace DA, Fridsma DB, et al. Identifying modifiable barriers to medication error reporting in the nursing home setting. J Am Med Dir Assoc. 2007;8:568-74.

15. Williams SD, Phipps DL, Ashcroft DM. Understanding the attitudes of hospital pharmacists to reporting medication incidents: a qualitative study. Res Soc Adm Pharm. 2013;9:80-9.

16. Ulanimo VM, O'Leary-Kelley C, Connolly PM. Nurses' perceptions of causes of medication errors and barriers to reporting. $\mathrm{J}$ Nurs Care Qual. 2007;22:28-33.

17. Malik MR, Alam AY, Mir AS, Malik GM, Abbas SM. Attitudes and perceived barriers of tertiary level health professionals towards incident reporting in Pakistan. N Am J Med Sci. 2010;2:100-5.

18. Evans SM, Berry JG, Smith BJ, Esterman A, Selim P, O'Shaughnessy J, et al. Attitudes and barriers to incident reporting: a collaborative hospital study. Qual Saf Heal Care. 2006;15:39-433.

19. Jeffe DB, Dunagan WC, Garbutt J, Burroughs TE, Gallagher $\mathrm{TH}$, Hill PR, et al. Using focus groups to understand physicians' and nurses' perspectives on error reporting in hospitals. Jt Comm J Qual Saf. 2004;30:471-9.

20. Kingston MJ, Evans SM, Smith BJ, Berry JG. Attitudes of doctors and nurses towards incident reporting: a qualitative analysis. MJA. 2004;181:36-9.

21. Walker SB, Lowe MJ. Nurses' views on reporting medication incidents. Int J Nurs Pract. 1998;4:97-102.

22. Sanghera IS, Franklin BD, Dhillon S. The attitudes and beliefs of healthcare professionals on the causes and reporting of medication errors in a UK Intensive care unit. Anaesthesia. 2007;62:53-61.

23. Samsiah A, Othman N, Jamshed S, Hassali MA. Perceptions and attitudes towards medication error reporting in primary care clinics: a qualitative study in Malaysia. PLoS ONE. 2016;11:e0166114.

24. Patrician PA, Brosch LR. Medication error reporting and the work environment in a military setting. J Nurs Care Qual. 2009;24:277-86.

25. Chiang H-Y, Lin S-Y, Hsu S-C, Ma S-C. Factors determining hospital nurses' failures in reporting medication errors in Taiwan. Nurs Outlook. 2010;58:17-25.

26. Aboshaiqah AE. Barriers in reporting medication administration errors as perceived by nurses in Saudi Arabia. Middle-East J Sci Res. 2013;17:130-6.

27. Sarvadikar A, Prescott G, Williams D. Attitudes to reporting medication error among differing healthcare professionals. Eur J Clin Pharmacol. 2010;66:843-53.

28. Carandang RR, Resuello D, Hocson GB, Respicio KM, Reynoso C. Knowledge, attitude and practices on medication error reporting among health practitioners from hospitals in Manila. Sch Acad J Pharm. 2015;4:293-9.

29. World Health Organization. Reporting and learning systems for medication errors: the role of pharmacovigilance centres. Geneva; 2014.

30. NPSF. RCA 2 Improving Root Cause Analyses and Actions to Prevent Harm. Boston, MA; 2016.

31. Stewart D, Thomas B, MacLure K, Wilbur K, Wilby K, Pallivalapila A, et al. Exploring facilitators and barriers to medication error reporting among healthcare professionals in Qatar using the theoretical domains framework: a mixed-methods approach. PLoS ONE. 2018;13:e0204987.

32. Alsulami SL, Sardidi HO, Almuzaini RS, Alsaif MA, Almuzaini HS, Moukaddem AK, et al. Knowledge, attitude and practice on medication error reporting among health practitioners in a tertiary care setting in Saudi Arabia. Saudi Med J. 2019;40:246-51.

33. Hollnagel E, Wears RL, Braithwaite J. From Safety-I to Safety-II : A White Paper. The Resilient Health Care Net: Published simultaneously by the University of Southern Denmark, University of Florida, USA, and Macquarie University, Australia. 2015.

34. World Health Organization. WHO Draft Guidelines for Adverse Event Reporting and Learning Systems. Geneva; 2005.

35. Kousgaard MB, Joensen AS, Thorsen T. Reasons for not reporting patient safety incidents in general practice: a qualitative study. Scand J Prim Health Care. 2012;30:199-205.

36. Hickner J, Zafar A, Kuo G. Field test results of a new ambulatory care medication error and adverse drug event reporting systemMEADERS. Ann Fam Med. 2010;8:517-25.

37. Cavell GF, Burgess V, Oborne CA, Williams R, Colwill S. Attitudes of community pharmacists towards medication error reporting. Int J Pharm Pract. 2001;9:64.

38. Kennedy AG, Littenberg B. Medication error reporting by community pharmacists in Vermont. J Am Pharm Assoc. 2004;44:434-9.

39. Winsvold Prang I, Jelsness-Jørgensen L-P. Should I report? A qualitative study of barriers to incident reporting among nurses working in nursing homes. Geriatr Nurs. 2014;35:441-7.

40. Dyab E, Elkalmi R, Bux S, Jamshed S. Exploration of nurses' knowledge, attitudes, and perceived barriers towards medication error reporting in a tertiary health care facility: a qualitative approach. Pharmacy. 2018;6:120.

41. Waaseth M, Ademi A, Fredheim M, Antonsen MA, Brox NMB, Lehnbom EC. Medication errors and safety culture in a Norwegian Hospital. Stud Health Technol Inform. 2019;265:107-12.

42. Teoh BC, Alrasheedy AA, Hassali MA, Tew MM, Samsudin MA. Perceptions of doctors and pharmacists towards medication error reporting and prevention in Kedah, Malaysia : A Rasch model analysis. Pharmacoepidemiol Adv Saf, Drug. 2015;4:192.

43. Ashcroft DM, Cooke J. Retrospective analysis of medication incidents reported using an on-line reporting system. Pharm World Sci. 2006;28:359-65.

44. Milch CE, Salem DN, Pauker SG, Lundquist TG, Kumar S, Chen J. Voluntary electronic reporting of medical errors and adverse events: an analysis of 92,547 reports from 26 acute care hospitals. J Gen Intern Med. 2006 Feb;21:165-70.

45. Cousins DH, Gerrett D, Warner B. A review of medication incidents reported to the National Reporting and Learning System in England and Wales over 6 years (2005-2010). Br J Clin Pharmacol. 2012;74:597-604.

46. Santell JP, Hicks RW, McMeekin J, Cousins DD. Medication errors: experience of the United States Pharmacopeia (USP) MEDMARX reporting system. J Clin Pharmacol. 2003;43:760-7.

47. Pierson S, Hansen R, Greene S, Williams C, Akers R, Jonsson M, et al. Preventing medication errors in long-term care: results and evaluation of a large scale web-based error reporting system. Qual Saf Health Care. 2007;16:297-302. 
48. Samsiah A, Othman N, Jamshed S, Hassali MA, Wan-Mohaina W. Medication errors reported to the national medication error reporting system in Malaysia: a four-year retrospective review (2009 to 2012). Eur J Clin Pharmacol. 2016;72:1515-24.

49. Ashcroft DM, Morecroft C, Parker D, Noyce PR. Likelihood of reporting adverse events in community pharmacy: an experimental study. Qual Saf Heal Care. 2006;15:48-52.

50. Khalil H, Lee S. Medication safety challenges in primary care: nurses' perspective. J Clin Nurs. 2018;27:2072-82.

51. Coley KC, Pringle JL, Weber RJ, Rice K, Ramanujam R, Sirio $\mathrm{CA}$. Perceived barriers in using a region-wide medication error reporting system. J Patient Saf. 2006;2:39-44.

52. Kaldjian LC, Jones EW, Wu BJ, Forman-Hoffman VL, Levi BH, Rosenthal GE. Reporting medical errors to improve patient safety. Arch Intern Med. 2008;168:40-6.

53. Schectman JM, Plews-Ogan ML. Physician perception of hospital safety and barriers to incident reporting. Jt Comm J Qual Patient Saf. 2006;32:337-43.

54. Mrayyan MT, Shishani K, Al-Faouri I. Rate, causes and reporting of medication errors in Jordan: nurses' perspectives. J Nurs Manag. 2007;15:659-70.
55. Petrova E. Nurses'perceptions of medication errors in Malta. Nurs Stand. 2010;24:41-8.

56. Almutary HH, Lewis PA, Cc C, Ed MN. Nurses ' willingness to report medication. Qual Manag Health Care. 2012;21:119-26.

57. Tabak N, Fleishman S. The intention to report medication error. In: Medicine and Law. 2011. p. 363-82.

58. Mirsadeghi A, Pazokian M. Barriers to reporting medication errors in Iran: a systematic review. Int J Med Rev. 2015;2:317-21.

59. Halligan M, Zecevic A. Safety culture in healthcare: a review of concepts, dimensions, measures and progress. BMJ Qual Saf. 2011;20:338-43.

60. Ajri-Khameslou M, Aliyari S, Hosein Pishgooie A, JafariGolestan N, Farokhnezhad AP. Factors affecting reporting of nursing errors: a qualitative content analysis study. Ann Med Heal Sci Res. 2018;8:215-9.

Publisher's Note Springer Nature remains neutral with regard to jurisdictional claims in published maps and institutional affiliations. 